\title{
Contrast Enhancement of an Image using Fuzzy Logic
}

\author{
Sonal Sharma \\ Student of C.S.E Dept. \\ DAVIET College, Jalandhar (PB) India, 144001
}

\author{
Avani Bhatia \\ Asst. Professor, Dept. of CSE \\ DAVIET College, Jalandhar (PB) India, 144001
}

\begin{abstract}
Image enhancement plays a significant role in vision applications. Many techniques have been proposed so far for enhancing the images. It has been found that the most of the existing techniques are based upon the transform domain methods; which may introduce the color artefacts and also may reduce the intensity of the input remote sensing image. To overcome this problem a modified approach is introduced in this research work. The new integrated approach has the capability to enhance the contrast in digital images in efficient manner by using the modified fuzzy based enhancement algorithm. Modified fuzzy image enhancement has integrated image gradients with input image for image enhancement. After image enhancement using modified fuzzy based algorithms the color normalization has come in action to reduce color artefacts. In order to evaluate the significant improvement of the proposed various well known images has been selected for experimental results. The experimental results have shown that the proposed technique has quite effective improvement over the available techniques.
\end{abstract}

\section{Keywords}

CD, CII ,EMEE, ME, Fuzzy enhancement.

\section{INTRODUCTION}

Image enhancement process consist of a collection of techniques that seek to improve the visual appearance of an image or to convert the image to a form better suited for analysis by a human or machine [1,2]. Image enhancement means as the improvement of an image appearance by increasing dominance of some features or by decreasing ambiguity between different regions of the image [2]. It is an indispensable tool for researchers in a wide variety of fields including medical imaging ,art studies, forensics and atmospheric sciences.It is application specific i.e an Image Enhancement technique suitable for one problem might be inadequate for another[3].

The objective of enhancement is to process an image so that the result is more suitable than the original image for a specific application[1,4].Contrast enhancements improve the perceptibility of objects in the scene by enhancing the brightness difference between objects and their backgrounds $[5,6]$. Contrast enhancements are typically performed as a contrast stretch followed by a tonal enhancement, although these could both be performed in one step. A contrast stretch improves the brightness differences uniformly across the dynamic range of the image, whereas tonal enhancements improve the brightness differences in the shadow (dark), mid tone (grays), or highlight (bright) regions at the expense of the brightness differences in the other regions[5].

\section{LITERATURE SURVEY}

G. Maragatham [7] proposed an algorithm to model images using its local contrast measure to classify and distinguish between the images having different contrast level. The input image is classified either as low contrast or high contrast image using the model. If the classified image is low contrast it is enhanced using the Stochastic Resonance principle. The results show that the proposed automated procedure enhances the low contrast image better than the conventional enhancement methods.

Thien Huynh- The and Thuong Le-Tien[8] an effective method Brightness Preserving Weighted Dynamic Range Histogram Equalization (BPWDRHE) has been proposed.It uses the weighted within-class variance as the new approach in separating out an original histogram The results prove that the BPWDHE method is better than the other methods in many quantitative measures, such as the overall brightness, the discrete entropy and the local contrast.

Yingjie Zhang[9] proposed a novel algorithm for image enhancement and denoising based on anisotropic diffusion and fuzzy logic theory. The maximum fuzzy entropy principle is used to map the normalized image to the fuzzy domain. Then, the image is filtered by the fractional anisotropic diffusion process iteratively. Hasikin K [10] Fuzzy grayscale enhancement technique for low contrast image is proposed by maximizing fuzzy measures contained in the image. The membership function is then modified to enhance the image by using power-law transformation and saturation operator. The proposed method produced better quality enhanced image and required minimum processing time compared to other methods.

M. Abdullah-Al-Wadud [12] A user-specified histogram equalization (UHE) approach, which provides user with a single variable to have the control over the enhancement procedure. By tuning this single parameter, user can achieve the desired enhanced look of the intended objects in an image is developed.

Li and Yang [14] have demonstrated an efficient way of contrast enhancement based on the fuzzy relaxation technique with improved speed and quality. Different orders of fuzzy mem-bership functions were tried out by various researchers in order to improve the speed and quality of contrast enhancement based on the fuzzy logic method. In the fuzzy method [9] gray tone is modelled into a fuzzy set using a membership function.Here the image is considered as an array of fuzzy singletons having a membership value that denotes the degree of some image property in the range. Applying an intensification operator globally modifies the membership function. Perona, P., And Malik, J. [11] proposed a new scalespace and edge detection algorithm using anisotropic diffusion method. In their technique, the diffusion coefficient is used in such a way to support the intra-region smoothing as comparative to inter-region smoothing,so as to extract the global information after removing the noise from the mage. This method does not necessitate any comparison of images at different scales, as the shape and position are preserved at every single scale and preserves the edge junctions. Dileep $\mathrm{MD}$ [13] A novel algorithm for image enhancement and denoising based on anisotropic diffusion and fuzzy logic theory is proposed. The maximum fuzzy entropy principle has been used to map the normalized image to the fuzzy domain. Then, the image was filtered by the fractional anisotropic 
diffusion process. The experimental results proved that the proposed method have removed noise and enhance contrast sufficiently and fine details of bore scope images were well preserved. The good performance of the proposed approach was due to the following factors: the maximum fuzzy entropy principle have made the proposed approach to handle the fuzziness of the images well; the S-function had enhanced the images without over-enhancement; and the sub pixel fractional diffusion that have removed noise and preserve the edges and details of the images well.

In the fuzzy method [15] gray tone is modelled into a fuzzy set using a membership function. Here the image is considered as an array of fuzzy singletons having a membership value that denotes the degree of some image property in the range. Applying an intensification operator globally modifies the membership function.

\section{PROPOSED METHODOLOGY}

In this section a new integrated approach is introduced which has the capability to enhance the contrast in digital images in efficient manner by using the modified fuzzy based enhancement algorithm. Modified fuzzy image enhancement will integrate image gradients with input image for image enhancement. After image enhancement using modified fuzzy based algorithms the color normalization will come in action to reduce color artefacts. The selection of the color normalization seems to be justifiable as it has the ability to overcome the problem of color artefacts in the existing methods.

Step 1: Take an input image.

Step 2: The colour of image is identified. If it is gray scale image then image gradient is applied on the image.An image gradient is a directional change in the intensity or color in an image. Image gradients are used to extract information from images.

Step 3: If the image is coloured then it is divided into three channels i.e Red,Green and Blue.Then image gradient is applied on each channel.

Step 4: After applying image gradients on the image Fuzzy based enhancement of image is done which is followed by 3 steps i.e Fuzzification, Membership modification, Defuzzification.

Step 5: After applying Fuzzy Enhancement on an image all the channels of a coloured image will be merged to get a full colour image.

Step 6: After concatenation Colour normalization of an image is done.It helps in removing shadows and thus producing a good quality image.

This section contains the algorithm for Proposed Fuzzy Enhancement. The algorithm is able to preserve edges in an image and removing the artefacts in an image.

Input: Image I, $\mathrm{x}$ and $\mathrm{y}$ are dimensions of the image I,

\section{Output: F}

$$
\begin{aligned}
& \text { f_lt }=5 \text {; } \\
& \text { fmt }=f \_l t(1)-\operatorname{abs}\left(-\mathrm{f} \_l t(1): f \_l t(1)\right) \text {; }
\end{aligned}
$$

Where $\mathrm{t}$ stands for Fuzzy limits and fmt stands for fuzzy membership type

2. To Develop Histogram

$$
[\mathrm{ch}, \mathrm{gs}]=\mathrm{I} \operatorname{Hist}(\mathrm{I}(\mathrm{x}, \mathrm{y}))
$$

Where ch stands for crisp histogram, gs stands for grayscale values and IHist for image histogram.

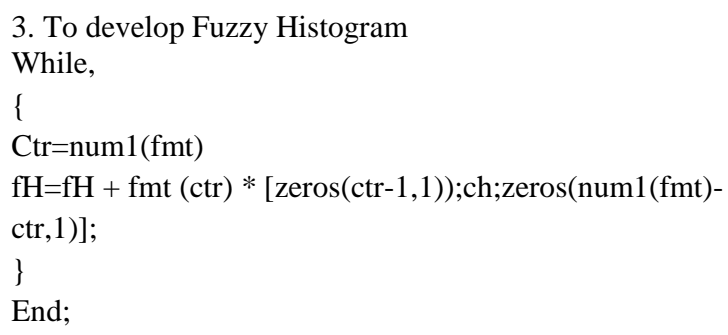
stands for Fuzzy membership type and num1 stands for first number.

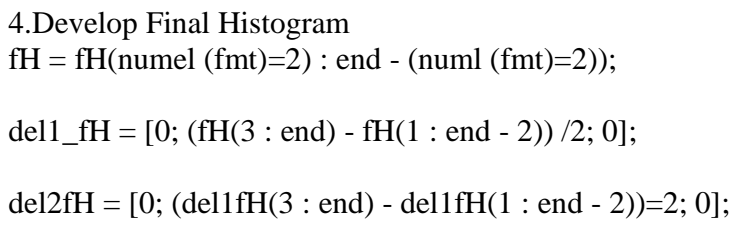

Where gs=grayscale values, tMap $=$ Transformation map

7. Evaluate Fuzzy Transformation map 
While

\{

ctr $\leq 1$ (low)

while $\{$ index $\leq$ high(ctr)

$\operatorname{tm}($ index $)=\operatorname{round}((\operatorname{low}(\mathrm{ctr})-1)$

(range $(\mathrm{ctr}) *(\operatorname{sum}($ fHist(low(ctr):index $))) /$

$(\operatorname{sum}(\operatorname{fHist}(\operatorname{low}(\mathrm{ctr}): \operatorname{high}(\mathrm{ctr}))))))$; \} \}

end

end

\section{Gradient Optimization}

epsilon = 0:000001;

$\mathrm{dt}=0: 1 ;[\mathrm{Ix}, \mathrm{Iy}]=\operatorname{grd}(\operatorname{grd}((\mathrm{F}(\mathrm{x} ; \mathrm{y}))))$;

$\mathrm{c}=1: /\left(\operatorname{sqrt}\left(\mathrm{Ix}^{2}+\mathrm{Iy}^{2}\right)+\right.$ epsilon $)$;

[div1; div2] $=\operatorname{grd}(\operatorname{grd}(\mathrm{c}: * \mathrm{Ix}))$;

[div3; $\operatorname{div} 4]=\operatorname{grd}(\operatorname{grd}(\mathrm{c}: * \operatorname{Iy}))$;

$\operatorname{div}=\operatorname{div} 1+\operatorname{div} 4$

$\mathrm{F}(\mathrm{x} ; \mathrm{y})=\mathrm{F}(\mathrm{x} ; \mathrm{y})-(\mathrm{dt}: * \mathrm{div})$;

where grd stands for gradient

\section{Color Normalization}

$\mathrm{Kr}=255 / \max (\max ((\mathrm{F}(\mathrm{X} ; \mathrm{Y})(: ;: ; 1))))$;

$\mathrm{Kg}=255 / \max (\max ((\mathrm{F}(\mathrm{X} ; \mathrm{Y})(: ; ; ; 2))))$;

$\mathrm{Kb}=255 / \max (\max ((\mathrm{F}(\mathrm{X} ; \mathrm{Y})(: ; ; ; 3))))$;

$\mathrm{F}(: ; ; ; 1)=\mathrm{Kr} *(\mathrm{~F}(\mathrm{X} ; \mathrm{Y})(: ; ; ; 1))$;

$\mathrm{F}(: ; ; ; 2)=\mathrm{Kg} *(\mathrm{~F}(\mathrm{X} ; \mathrm{Y})(: ; ; ; 2))$;

$\mathrm{F}(: ; ; ; 3)=\mathrm{Kb} *(\mathrm{~F}(\mathrm{X} ; \mathrm{Y})(: ; ; ; 3))$;

\section{EXPERIMENTAL RESULTS}

In the experiments, the proposed approach has been implemented to test a wide variety of images. The algorithm has been implemented in MATLAB. In this section, we have compared existing fuzzy based image enhancement [10] with our proposed algorithm showing their comparison through images. Then technique is compared on the basis of values generated by various parameters.

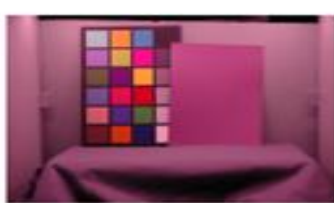

(a) Wall Frame

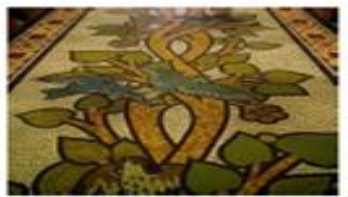

(c) Painting

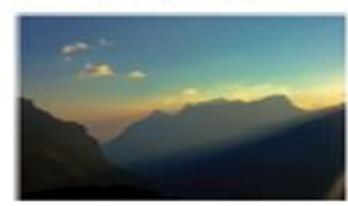

(e) Hills

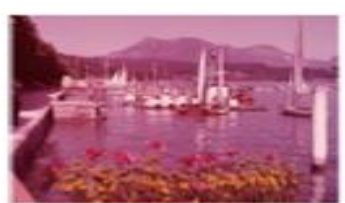

(b) Lake

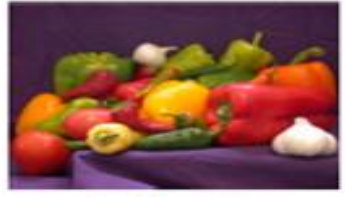

(d) Vegetables

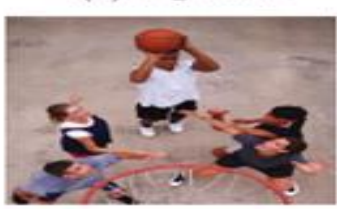

(f) Basketball Players
Fig1: Input Images (a) Wall Frame (b) Lake (c) Painting (d) Vegetables (e) Hills (f) Basketball Players

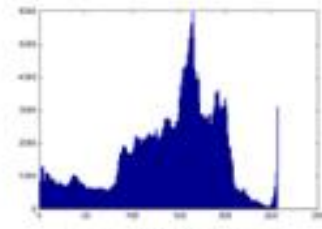

(a) Wall Frame

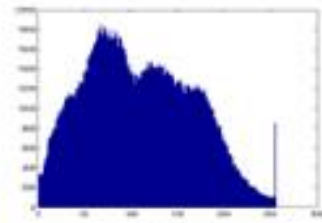

(c) Painting

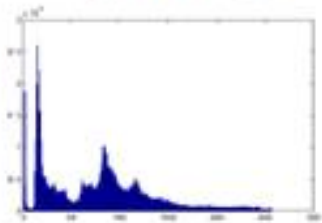

(e) Hills

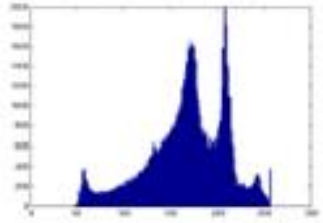

(b) Lake

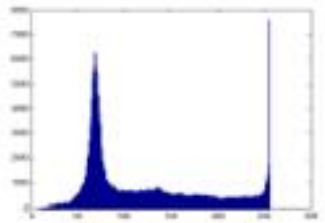

(d) Vegetables

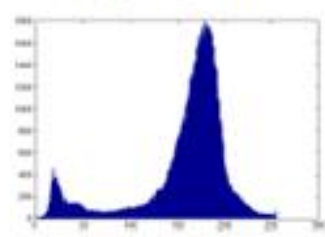

(f) Basketboll Players
Figure 2: Histogram of Input Images (a) Wall Frame (b) Lake (c) Painting (d) Vegetables (e) Hills (f) Basketball Players

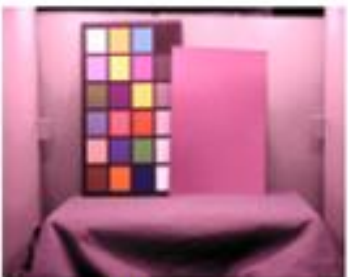

(a) Wall Frame

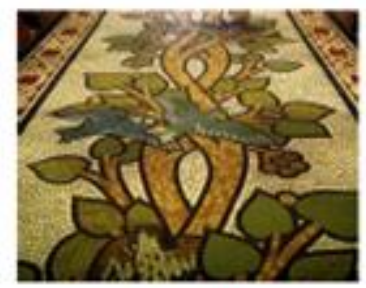

(c) Painting

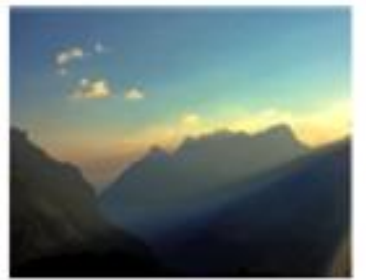

(e) Hills

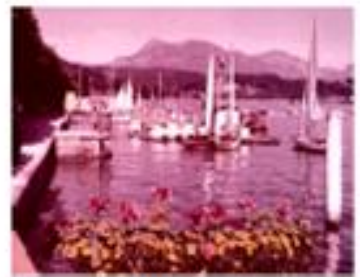

(b) Lake

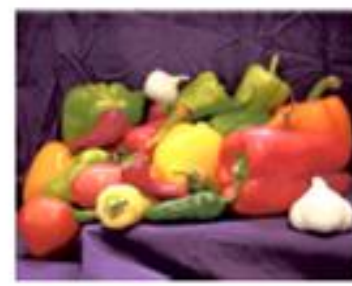

(d) Vegetables

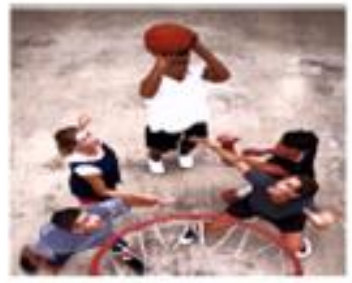

(f) Baskotball Players
Figure 3 :Images after applying Existing Fuzzy

Enhancement Technique[16] (a) Wall Frame (b) Lake (c)Painting (d) Vegetables (e) Hills (f) Basketball Players 


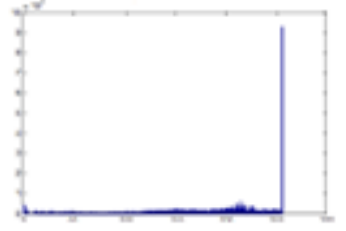

(a) Wall Frame

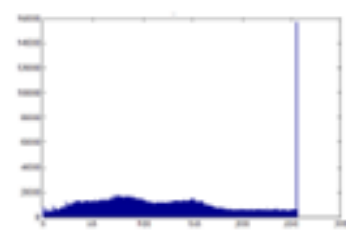

(c) Painting

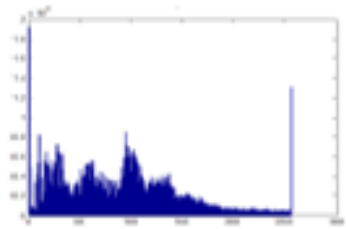

(e) Hills

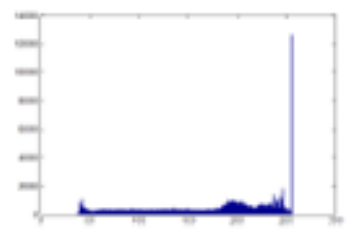

(b) Lake

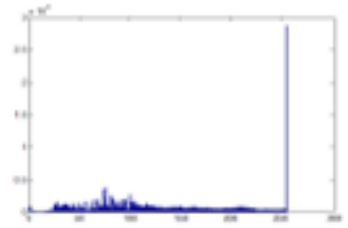

(d) Vegetables

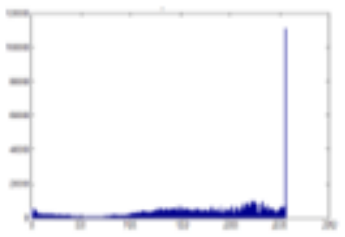

(f) Basketball Players
Figure 4 : Histogram of Images after applying Existing Fuzzy Enhancement Technique[16] (a) Wall Frame (b) Lake (c)Painting (d) Vegetables (e) Hills (f) Basketball Players

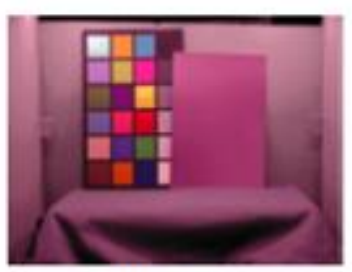

(a) Wall Frame

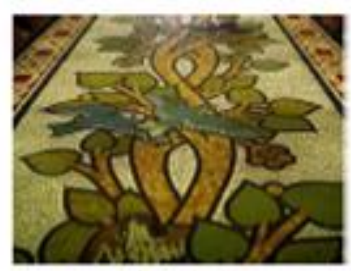

(c) Painting

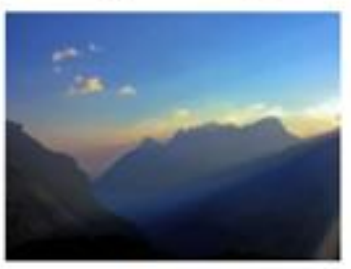

(e) Hills

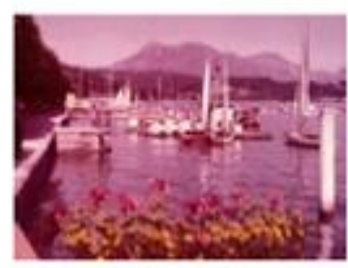

(b) Lake

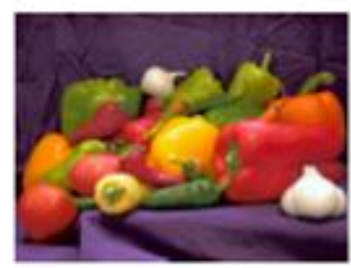

(d) Vegetables

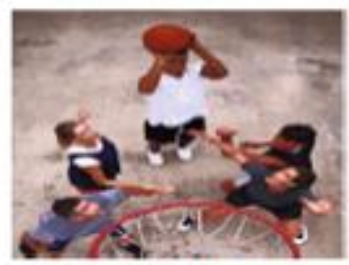

(f) Basketball Players

Figure 5 : Images after applying Proposed Technique (a) Wall Frame (b) Lake (c)Painting (d) Vegetables (e) Hills (f) Basketball Players.

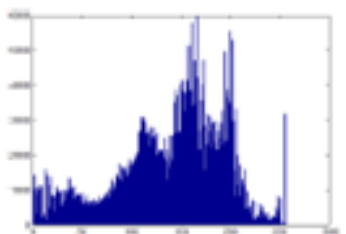

(a) Wall Frame

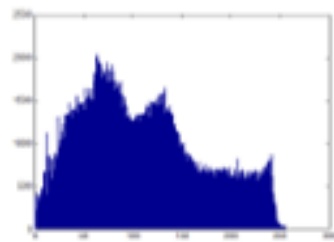

(c) Painting

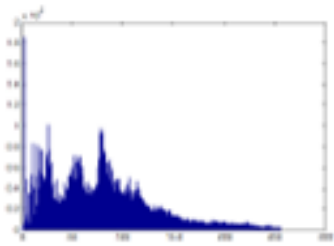

(e) Hills

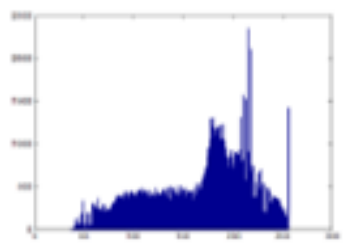

(b) Lake

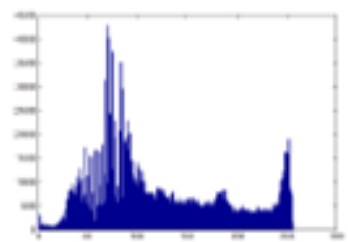

(d) Vegetables

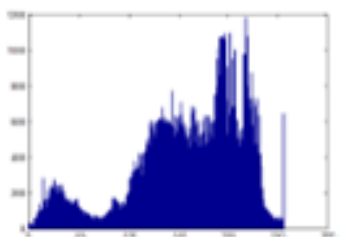

(f) Basketball Players
Figure 6 : Histogram of Images after applying Proposed Technique (a) Wall Frame (b) Lake (c)Painting (d) Vegetables (e) Hills (f) Basketball Players

The performance measure values shows that the proposed method gives better results compared to the conventional and advanced enhancement techniques.

\section{A) Contrast Difference (CD):}

Contrast difference is the difference between maximum (Imax) and minimum (Imin) pixel intensity of an image. High value of contrast difference stands for enhancing brighter (high gray level) pixel to brightest and darker (pixel with low value) to darkest pixel[16].It is defined as;

$$
\mathrm{CD}=\operatorname{Imax}-\operatorname{Imin}
$$

Table 1 has shown the comparison among proposed and the existing strategy based on Contrast Difference. The graph obtained for Contrast Difference of these images is shown in figure 7 below:

Table1: CD Analysis

\begin{tabular}{|l|l|l|}
\hline Image & $\begin{array}{l}\text { Existing } \\
\text { Technique[16] }\end{array}$ & $\begin{array}{l}\text { Proposed } \\
\text { Technique }\end{array}$ \\
\hline IMG 1 & 0.1342 & 0.4395 \\
\hline IMG 2 & 0.1913 & 2.3078 \\
\hline IMG 3 & 0.7284 & 4.1480 \\
\hline IMG 4 & 0.1651 & 3.0094 \\
\hline IMG 5 & 0.0628 & 7.6090 \\
\hline IMG 6 & 0.2026 & 0.5029 \\
\hline IMG 7 & 0.4087 & 3.8035 \\
\hline IMG 8 & 0.1337 & 0.6948 \\
\hline IMG 9 & 0.1184 & 7.7292 \\
\hline IMG 10 & 0.1955 & 11.4909 \\
\hline
\end{tabular}




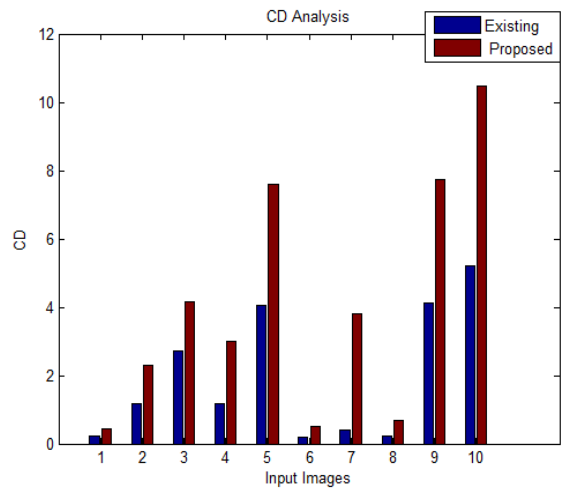

Figure 7: CD Analysis

\section{B) Measure of Contrast Improvement Index (CII)}

This metric is defined as the ratio of enhanced contrast (Cenhanced) to the original contrast (Coriginal). A higher value of contrast improvement index is always preferred. It is formulated as shown below[16]. Table 2 has shown the comparison among proposed and the existing strategy based on Contrast Improvement Index. As the value CII is more in almost every taken image; therefore the proposed strategy has shown significant results over the available technique. The graph obtained for Contrast Improvement Index of these images is shown in figure 8 below:

Table2: CII Analysis

\begin{tabular}{|l|l|l|}
\hline Image & $\begin{array}{l}\text { Existing } \\
\text { Technique[16] }\end{array}$ & $\begin{array}{l}\text { Proposed } \\
\text { Technique }\end{array}$ \\
\hline IMG 1 & 1.0047 & 1.4628 \\
\hline IMG 2 & 1.0051 & 1.3843 \\
\hline IMG 3 & 1.0064 & 1.3912 \\
\hline IMG 4 & 1.0083 & 1.5762 \\
\hline IMG 5 & 1.0073 & 1.5208 \\
\hline IMG 6 & 1.0071 & 1.5049 \\
\hline IMG 7 & 1.0062 & 1.4801 \\
\hline IMG 8 & 1.0055 & 1.3722 \\
\hline IMG 9 & 1.0085 & 1.4953 \\
\hline IMG 10 & 1.0058 & 1.3713 \\
\hline
\end{tabular}

The graph obtained for Contrast Difference of these images is shown in figure 8 below:

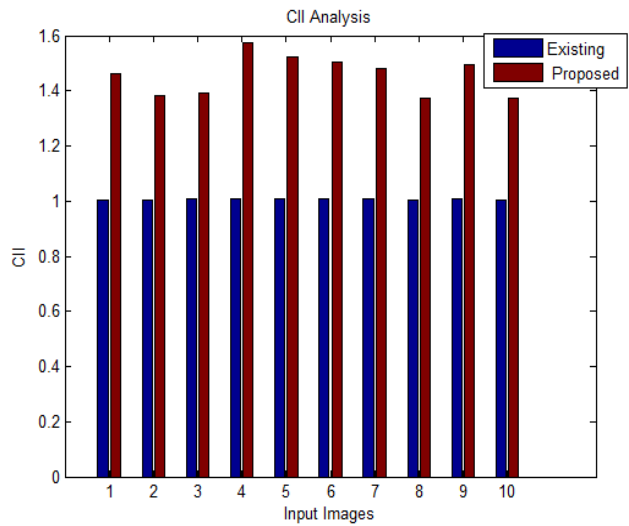

Figure 8: CII Analysis

\section{C) Measure of Entropy (ME)}

This metric is a statistical measure of randomness used to characterize the texture of an image. The entropy is calculated by using Shannon's entropy theorem. Whenever the entropy is high it is clear that the image has high contrast[16]. Table 3 has shown the comparison among proposed and the existing strategy based on Measure of Entropy. As the ME is more in almost every taken image.

Table3: ME Analysis

\begin{tabular}{|l|l|l|}
\hline Image & $\begin{array}{l}\text { Existing } \\
\text { Technique[16] }\end{array}$ & $\begin{array}{l}\text { Proposed } \\
\text { Technique }\end{array}$ \\
\hline IMG 1 & 3.5455 & 7.5822 \\
\hline IMG 2 & 3.7230 & 7.8731 \\
\hline IMG 3 & 3.8109 & 7.6708 \\
\hline IMG 4 & 3.2979 & 7.3732 \\
\hline IMG 5 & 3.7008 & 7.4645 \\
\hline IMG 6 & 3.0324 & 7.5577 \\
\hline IMG 7 & 3.7643 & 7.8192 \\
\hline IMG 8 & 3.4475 & 7.2078 \\
\hline IMG 9 & 3.4335 & 7.5996 \\
\hline IMG 10 & 3.2035 & 7.5856 \\
\hline
\end{tabular}

The graph obtained for Measure of Entropy of these images is shown in figure 9 below:

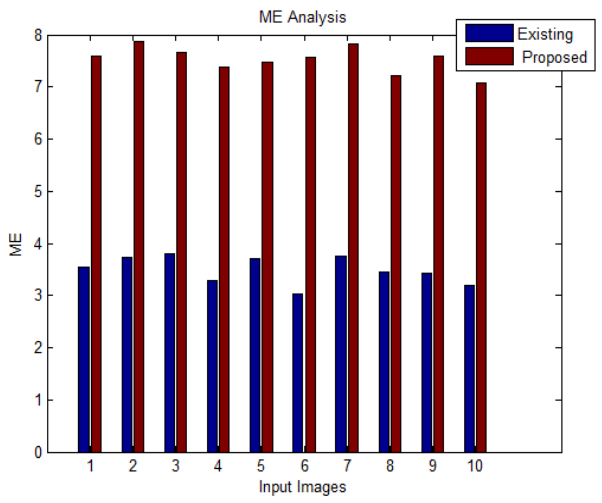

Figure 9: ME Analysis

\section{D) Measure of enhancement by entropy (EMEE):}

The metric entropy is defined as the average of ratio of maximum pixel intensity Imax to the Imin intensities in decibel and maximum and minimum intensities of enhanced image( Iemax, ,Iemin ). This metric does not show human perception but measures the local contrast. For a high

contrast image, enhanced entropy will be high[16].Table 4 has shown the comparison among proposed and the existing strategy based on EMEE. As the value of EMEE is more in almost every taken image therefore our proposed strategy is better than the existing one.

Table4: EMEE Analysis

\begin{tabular}{|l|l|l|}
\hline Image & $\begin{array}{l}\text { Existing } \\
\text { Technique[16] }\end{array}$ & Proposed Technique \\
\hline IMG 1 & 5.8803 & 14.6556 \\
\hline IMG 2 & 7.5285 & 15.6384 \\
\hline IMG 3 & 6.7885 & 14.1374 \\
\hline
\end{tabular}




\begin{tabular}{|l|l|l|}
\hline IMG 4 & 6.8136 & 15.0809 \\
\hline IMG 5 & 6.4070 & 15.1713 \\
\hline IMG 6 & 7.4016 & 14.8707 \\
\hline IMG 7 & 5.1546 & 11.1012 \\
\hline IMG 8 & 7.4170 & 15.6847 \\
\hline IMG 9 & 6.9275 & 14.8147 \\
\hline IMG 10 & 5.7774 & 12.9167 \\
\hline
\end{tabular}

The graph obtained for Measure of Enhancement by Entropy of these images is shown in figure 10

Below:

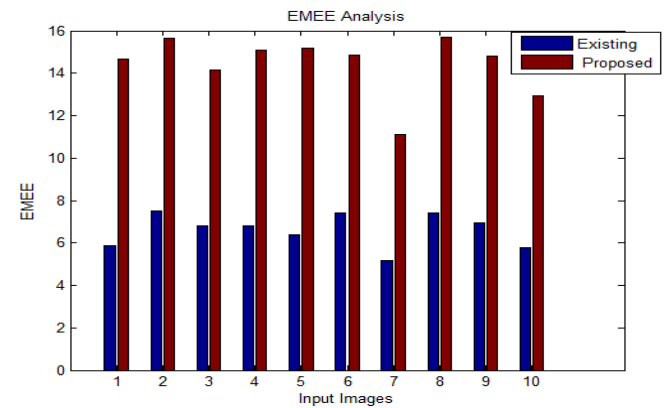

Figure 10: EMEE Analysis

\section{E) Measure of Luminance Index (MLI)}

This metric is defined as the ratio of mean (MI) of enhanced image Ie to the mean of original image Io. In this case Luminance index is used as a measure of intensity. For a good quality image MLI must be of high value[16].

Table 5 has shown the comparison among proposed and the existing strategy based on MLI.As the value of MLI is more in almost every taken image therefore our proposed strategy is better than the existing one. The graph obtained for Measure of Enhancement by Entropy of these images is shown in figure 10 below.

Table5: MLI Analysis

\begin{tabular}{|l|l|l|}
\hline Image & $\begin{array}{l}\text { Existing } \\
\text { Technique[16] }\end{array}$ & $\begin{array}{l}\text { Proposed } \\
\text { Technique }\end{array}$ \\
\hline IMG 1 & 0.8908 & 0.9870 \\
\hline IMG 2 & 0.8893 & 0.9898 \\
\hline IMG 3 & 0.9662 & 0.9958 \\
\hline IMG 4 & 0.9138 & 0.9986 \\
\hline IMG 5 & 0.7714 & 0.9996 \\
\hline IMG 6 & 0.9687 & 0.9935 \\
\hline IMG 7 & 0.9546 & 0.9987 \\
\hline IMG 8 & 0.9944 & 0.9960 \\
\hline IMG 9 & 0.8363 & 0.9581 \\
\hline IMG 10 & 0.8677 & 0.9996 \\
\hline
\end{tabular}

Figure 11: MLI Analysis

\section{CONCLUSION AND FUTURE SCOPE}

Aim of image enhancement is to improve the interpretability or perception of information in images for human viewers .Fuzzy techniques can manage the vagueness and ambiguity efficiently and provides a capability to characterize the uncertainty and imprecision and to incorporate human knowledge into problem solving process. To overcome this problem a modified approach is introduced in this research work. The new integrated approach has the capability to enhance the contrast in digital images in efficient manner by using the modified fuzzy based enhancement algorithm. Modified fuzzy image enhancement has integrated image gradients with input image for image enhancement. After image enhancement using modified fuzzy based algorithms the color normalization has come in action to reduce color artefacts. The selection of the color normalization seems to be justifiable as it has the ability to overcome the problem of color artefacts in the existing methods. In order to evaluate the significant improvement of the proposed various well known images has been selected for experimental results. The proposed technique has been designed and implemented in the MATLAB tool with the help of image processing and data analysis toolbox. The experimental results has shown that the proposed technique has quite effective improvement over the available techniques. Various kinds of quality metrics for objective evaluation has also been used to compare the proposed technique with the existing methods.

This work has not considered any optimization technique to find the optimistic adjustment function to improve the image enhancement further. Also the effect of the noise on the input images has also been neglected in the this paper. So in near future two improvements can be done. Firstly one can use the different kind of optimization techniques to find the optimistic adjustment function. Secondly one can proposed filtering based hybrid image enhancement to improve the image enhancement further.

\section{REFERENCES}

[1] Vijay A. Kotkar and Sanjay S. Gharde "Review of various Image Contrast Enhancement Techniques", International Journal of Innovative Research in Science, Engineering and Technology,Vol. 2, No. 7, July 2013.

[2] Vijay Dhir and Sanjeev Kumar "Review of Various Image Contrast Enhancement Techniques",International Journal of Advanced Research in Computer Science and Software Engineering,Vol.4,No.8,August 2014.

[3] Hanan Saleh S. Ahmed and Md Jan Nordin "Improving Diagnostic Viewing of Medical Images using Enhancement Algorithms ", Journal of Computer Science vol.7,No.12,2011.

[4] Raman Maini and Himanshu Aggarwal "A Comprehensive Review of Image Enhancement Techniques ", Journal of Computing , vol.2, No.3, March 2010.

[5] Tarun Dewangan, M.A.Siddiqui and RCET Bhiali "Analysis of Contrast Enhancement Method Using Modi_ed Dynamic Histogram ", International Journal of Engineering Science and Innovative Technology (IJESIT), Volume 2, No. 3, May 2013.

[6] Arun Kavi Arasu.S, Mohamed Nizar.S and Prabakaran.D "Review of Image Contrast Enhancement Techniques ",International Journal of Engineering Research \& Technology (IJERT),Vol.2,No.7, November 2013. 
[7] G. Maragatham, S.Md.Mansoor Roomi "An Automatic Contrast Enhancement method based on Stochastic Resonance ", In IEEE Fourth International Conference on Computing, Com munications and Networking Technologies (ICCCNT), Tiruchengode,India , pp.1-7, July 2013.

[8] Thien Huynh- The,Thuong Le-Tien "Brightness Preserving Weighted Dynamic Range Histogram Equalization for Image Contrast Enhancement ",In IEEE International Conference on Advanced Technologies for Communications (ATC'13), pp.386-391, 2013.

[9] Yingjie Zhang "A Novel Contrast Enhancement and Denoising Method for Borescope Images ", In IEEE 5fth International Conference on Advanced Computational Intelligence( ICACI) , pp.570-573,October 2012.

[10] Khairunnisa Hasikin, Nor Ashidi Mat Isa "Enhancement of the low contrast image using fuzzy set theory ", In IEEE 14th International Conference on Modelling and Simulation , pp.371-376, 2012.

[11] P. Perona and J. Malik, "Scale-space and edge detection using anisotropic diffusion ", IEEE Trans. Pattern Anal. Mach. Intell vol.12,no. 7, pp 629-639, 1990
[12] M. Abdullah-Al-Wadud , Yoojin Chung "A Userspecified Approach for Image Contrast Enhancement ", 12th International Conference on Intelligent Systems Design and Applications (ISDA),pp.937-940,2012.

[13] Dileep MD, A.Sreenivasa Murthy “A Comparison between different Colour Image Contrast Enhancement Algorithms ", In IEEE International Conference on Emerging Trends in Electrical and Computer Technology (ICETECT), pp.708-712, 2011.

[14] Li H, Yang HS "Fast and reliable image enhancement using fuzzy relaxation technique ", IEEE Trans Syst Man Cybern vol.19,,pp.127681,1989.

[15] Pal SK, King RA.”Image enhancement using smoothing with fuzzy sets " IEEETrans Syst Man Cybern , pp.494501, July 7.

[16] Reshmalakshmi C. and Sasikumar M. Umage Con-trast Enhancement using Fuzzy Technique ",International Conference on Circuits, Power and Computing Technologies [ICCPCT-2013],pp.861-865,2013. 